\title{
¿DONDE ESTAN LAS OPORTUNIDADES PARA AGREGAR VALOR?
}

\author{
WHERE ARE THE OPPORTUNITIES TO ADD VALUE?
}

\author{
Jairo Omar Delgado Mora1,, Luz Mireya Pamplona Camargo²
}

\begin{abstract}
RESUMEN
La Agricultura en América Latina es la mejor opción que se vislumbra a futuro como fuente generadora de desarrollo. En ese sentido, identificar las oportunidades para agregar valor (AV), que surgen de la cadena de valor que constituye el proceso agrícola es el soporte para la realización de esta investigación. Hoy esa búsqueda de AV se hace indispensable para poder mantener vigencia, competitividad en el mercado. Es así como funcionan las diferentes formas de integración de las grandes organizaciones con los pequeños empresarios. De hecho, para el caso Latinoamericano es una de las mejores opciones para poder acceder al mercado global. De la revisión de la información documentada se han identificado cinco dimensiones a través de las cuales se puede AV dentro de un proceso productivo aplicados al caso de la agricultura.
\end{abstract}

Palabras Clave: Agregar valor, diferenciación, mejores prácticas, manufactura esbelta, competitividad.

\section{ABSTRACT}

Agriculture in Latin America is the best option that can be seen in the future as a source of development. In this sense, identify opportunities to add value (AV), which arise from the value chain that constitutes the agricultural process and are the support for the realization of this document. Today that search for AV is essential to maintain validity, competitiveness in the market. This is how the different forms of integration of large organizations work with small entrepreneurs. In fact, for the Latin American case, it is one of the best options to access the global market. From the review of the documented information, was made the identification of

\footnotetext{
1 Universidad pedagógica y tecnológica de Colombia; docente, Duitama, Colombia.

2Universidad pedagógica y tecnológica de Colombia; Licenciatura en educación Básica. Duitama, Colombia. luzmireya.pamplona@uptc.edu.co.

- Autor para correspondencia: jairo.delgado@uptc.edu.co 
five dimensions through which value can be AV within a production process on an application to the case of agriculture.

Keywords: Add value, differentiation, best practices, lean manufacturing, competitiveness.

\section{INTRODUCCION}

Agregar valor (AV) se ha convertido en un instrumento tan importante que es el corazón de las mejores prácticas de clase mundial (best practices) y es el motor de la manufactura esbelta (Liker, 2004), pues la manufactura esbelta surge como un instrumento para disminuir los desperdicios que se generan en los procesos de producción de bienes o servicios, para lo cual disgregar esos procesos, observarlos con microscopio, encontrar las causas a esos desperdicios es lo que involucra el termino $\mathrm{AV}$, el cual requiere establecer las forma a través de la cual se debe realizar esa observación detallada de los procesos, convirtiéndose en un factor diferenciador del mercado global de cualquier tipo de actividad; ambiental (Chávez \& Guarín, 2014), agrícola (Kudinova et al. 2012) por ejemplo. Incluso, este instrumento promueve grandes oportunidades para resolver problemas como el desempleo, inequidad y subdesarrollo de la mayoría de países Latinoamérica, como lo ha propuesto Kudinova et al. (2012). Agregar valor requiere hacer un análisis de interpretación de: fenómenos, resultados de indicadores, metas, reportes de monitoreo, mediciones realizadas, la misma observación del desempeño de los procesos, y a partir de ellos generar factores que diferencian un mismo proceso entre organizaciones. Este ejercicio de interpretación y toma de decisiones no puede ser realizado por una máquina, un instrumento sino por el ser humano. Por esta razón, el ser humano se constituye en la fuente inspiradora de $\mathrm{AV}$, y requiere especial atención, la manera como él mismo se convierte en la herramienta para encontrar la forma como en él se pueden detectar esas oportunidades de AV.

\section{MATERIAL Y METODOS}

Con base en lo anterior, el documento se ha construido sobre la base de una revisión de tipo cualitativo, pues se soporta en descripciones, mensajes, ideas (Ballen, 2007) plasmadas en los documentos consultados acerca del tema de agregar valor y dispuestos en las bases de datos internacionales siguiendo metodología de mapas conceptuales propuestos en Delgado (2017a).

Se consideraron la perspectiva humana tomando como base la investigación de Calderón (2008). Para las categorías donde se ha agregado valor en el ser humano, se consideró el trabajo de la Sociedad Bíblica Católica Internacional (1972). Ambos documentos fueron contrastados con lo reportado en Liker (2004). De esta revisión teórica, se ratificaron cinco dimensiones a través de las cuales se puede AV en una actividad, proceso, proyecto, producto, 
investigación, área funcional de una organización con base en los factores involucrados en el proceso de desarrollo agrícola establecidos por Restrepo (2015), Daniloska (2016) y Kudinova (2012). Se consideró en los análisis experiencia empírica sobre AV en las organizaciones con base en las investigaciones de Porter (2008) y de Chacón y García (2015).

\section{RESULTADOS Y DISCUSIÓN}

Atendiendo a los objetivos plantados, el primer aspecto a revisar tiene que ver con la forma como se ha planteado AV desde la perspectiva divina. Para tal efecto, el documento presentado por la Sociedad Bíblica Católica Internacional (1972), se constituye en el instrumento elegido.

\section{Las oportunidades de AV desde la deidad.}

El ser humano como factor diferenciador fundamental para AV, tiene incorporado, en su ser, una serie de recursos, enmarcados en las virtudes, que se hacen extensivos y se consolidan en el desarrollo organizacional en general.

En primera instancia, el ser humano tiene una necesidad de identificarse con algo, creer en algo, esto es lo que se reconoce como la alineación con una estrategia plasmada en ese sueño de alcanzar, o la esperanza de un mejor vivir (Teófilo, 2016). Es así como el ser como tal aparece inicialmente "desnudo" y posteriormente le son incorporados una serie de recursos básicos y en diferentes proporciones para que pueda ser diferenciado entre los de su misma especie y los otros seres vivos. Estas virtudes en sí, constituyen los primeros factores básicos que pueden servir como elementos a agregar valor y se pueden asociar con lo que hoy reconocemos como las herramientas de manufactura esbelta (Lean Manufacturing), que parten de su propia voluntad para hacerlo.

La segunda dimensión para agregar valor incorporado al ser, corresponde de acuerdo a Calderón (2008) a lo que se ha denominado, las características comunes y se manifiestan a través de los mecanismos de control que sugieren el andar por un camino correcto; los valores, buen uso del capital intelectual, agente de cambio, tienen que ver con el comportamiento personal y en sociedad, están muy ligados a la conciencia.

Una tercera dimensión para $\mathrm{AV}$, son las responsabilidades específicas y asignadas por la gerencia y que están encaminadas a desarrollar un proyecto de mejoramiento o de acción dentro de la misma organización.

Estas tres dimensiones mencionadas, requieren un fuerte componente de acompañamiento y despliegue proporcional de autoridad como ha sido reportado por Calderón (2008) para mantener un equilibrio, (cuarta dimensión de AV) dentro de ellas y entre ellas, como la autogestión (Druskat \& Wheeler, 2004) y que, como consecuencia de ello, aparezca un ser integral, idóneo para la organización y la sociedad, producto de la efectividad del mismo producto del monitoreo y medición (quinta dimensión para $\mathrm{AV}$ ) realizado a su desempeño. En la Figura 1, se identifican esas cinco oportunidades para AV. 


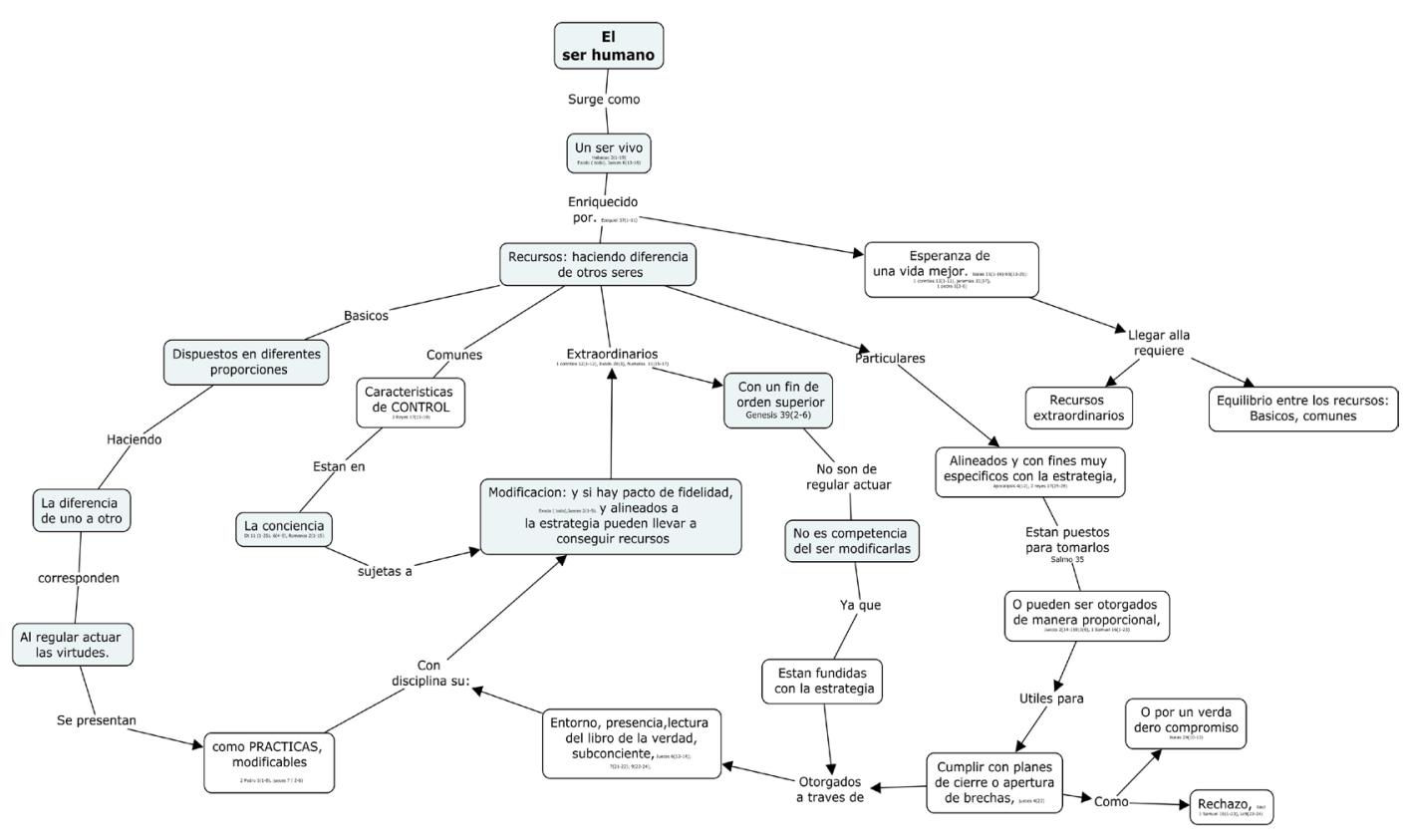

Figura 1. Oportunidades de agregación de valor.

\section{El valor agregado, perspectiva terrenal}

A continuación y conforme con lo establecido inicialmente, se hace la exploración de cuáles son las oportunidades de AV que se han establecido por parte de aquellos que han estudiado el tema, pero tomando una perspectiva diferente a la deidad.

El valor agregado surge como una herramienta de manufactura esbelta que promueve dos cosas en particular: reducción de desperdicios y el flujo de producción. (Liker, 2004; Mobley, 2014). En este sentido, dentro del desarrollo de un proceso pueden existir actividades que consumen recursos sin crear valor para el cliente, desequilibrio dentro o entre procesos, que igualmente generan sobrecostos, por ejemplo, en el manejo de inventarios, sobrecargas en el trabajo de equipos o personas para poder cumplir con unos requerimientos de los clientes. Surge entonces, entre otras, la siguiente inquietud, ¿Hasta que tanto se deben utilizar las herramientas de manufactura esbelta para ofrecer un producto o servicio capaz de entregar un producto que esté dispuesto el cliente a pagar?

En primer lugar, se requiere un compromiso por la dirección el cual se plasma en una estrategia que es desplegada y aceptada por los trabajadores, seleccionar una línea de producción o un proyecto para tomarlo como piloto para adaptar una herramienta de Lean escogida e implementada previamente (Rajadell, 2010), evaluar y controlar los resultados, buscando cerrar la brecha entre lo deseado y lo obtenido, promover disciplina, autogestión en los trabajadores, estandarizar, generar conocimiento producto de la lección aprendida, desplegar esto que se ha aprendido hacia las otras áreas funcionales y/o iniciar el mismo proceso con otra herramienta. (González, 2007; Liker, 2004; Delgado, 2015)

Con la estrategia definida, el enfoque de AV busca entonces identificar esos aspectos en los cuales hay problemas de calidad, desequilibrio dentro o entre procesos, tiempos muertos, disminución de tiempos de ciclo de trabajo de los operadores o de las máquinas para favorecer 
el flujo continuo de la producción, permitiendo incrementar la frecuencia de entregas internas y externas, mejorando la calidad y reubicando al personal hacia otras áreas. (González, 2007; Liker, 2004; Chybalski, 2017)

En esta perspectiva, se identifican las siguientes dimensiones a través de los cuales se puede agregar valor, sobre la base de una herramienta de lean seleccionada: mecanismos de control, definición de una actividad particular o piloto (de un proceso) para desarrollar el trabajo, personal previamente capacitado y entrenado para la realización de la intervención, búsqueda de equilibrio: dentro o entre las actividades y los métodos de monitoreo/medición para favorecer la gestión.

\section{Consolidación de las dos perspectivas seleccionadas para identificar las oportunidades de AV}

Atendiendo al deseo de confrontar las dos formas de caracterizar la forma de AV presentadas, a continuación se sintetizan esas dimensiones, destacando que la coincidencia que hay en las mismas. En este sentido, las dimensiones consolidadas son:

Mecanismos de control: Especificaciones de productos, conciencia del ser humano, las leyes que rigen el comportamiento de las personas en comunidad, información documentada de las organizaciones, rutinas de actuación en aspectos judiciales, quirúrgicos, protocolos clínicos. Las características propias del proceso: un instrumento, o equipo, específico para realizar una labor, unas cualidades del ser como la piedad, el amor fraterno, las características propias de un proceso/equipo quirúrgico, la forma como opera un área funcional de una organización.

Las competencias inherentes al ser humano; formación académica, habilidades, motivación, experiencia en la actividad a desarrollar. Es decir, saber, saber -hacer, querer-hacer, (Delgado, 2015).

Monitoreo y medición: estadística descriptiva, diagramas de control de procesos, encuestas de satisfacción, cálculo del valor presente neto, la determinación de la tasa interna de retorno, evolución de un paciente, impacto de una estrategia desplegada, determinación de la incertidumbre de un instrumento de medición.

Equilibrio: en o entre las actividades, las correcciones, acciones correctivas en los procesos productivos, el autocontrol, autogestión y autorregulación en los trabajadores, toma de decisiones judiciales, políticas, medicas.

De la abstracción realizada para las dos revisiones documentales realizadas; las oportunidades de AV desde la deidad y el valor agregado perspectiva terrenal, y dado que las dos son coincidentes en las dimensiones identificadas se concluye que estas siempre han estado presentes, pues así lo relata lo extractado desde el texto bíblico, el cual data de más de 2600 años de haber sido escrito. Pero que el ser humano lo puedo evidenciar solamente después del desarrollo suscitado como consecuencia de la segunda guerra mundial.

Igualmente, no sobra mencionar que dentro de estas revisiones realizadas se encuentran inmersos los conceptos de planear, hacer, verificar y actuar promulgados por Deming, al igual que lo referente a: eficiencia, eficacia que se consolidan en el concepto de efectividad.

Pero no menos importante que sumergir los procesos dentro del ciclo PHVA y con efectividad es relevante que cualquier acción encaminada a agregar valor, debe presentar soluciones satisfactorias en todo su contexto (Chybalski, 2017). Pues no sobra mencionar que hoy por hoy se desarrollan muchos proyectos dentro de este marco de PHVA y efectividad, cuyas 
soluciones no son del todo satisfactorias para todas las partes interesadas, por aquellas cosas de la corrupción implícitas en ellas, (Delgado, 2017b).

En el Tabla 1, se han colocado algunas experiencias documentadas a través de las cuales se ha realizado el ejercicio de agregar valor:

Tabla 1. Experiencias de agregación de valor.

\begin{tabular}{|l|l|l|}
\hline \multicolumn{1}{|c|}{ Tema documentado } & \multicolumn{1}{|c|}{$\begin{array}{c}\text { Dimensión donde se agrego } \\
\text { valor }\end{array}$} & Fuente \\
\hline $\begin{array}{l}\text { Heurística para el balance } \\
\text { de líneas de ensamble }\end{array}$ & $\begin{array}{l}\text { Mecanismos de control, monitoreo y } \\
\text { medición, equilibrio }\end{array}$ & $\begin{array}{l}\text { Chacón y García (2015) } \\
\text { Características propias del proceso } \\
\text { (nuevos participantes), equilibrio } \\
\text { (rivalidad entre competidores, poder } \\
\text { de los compradores y proveedores, } \\
\text { sustitución), mecanismos de } \\
\text { control (nuevos participantes), } \\
\text { monitoreo y medición (rivalidad } \\
\text { entre competidores), y por supuesto } \\
\text { todas ellas obligan a la dinámica del } \\
\text { ser enmarcada en las competencias } \\
\text { inherentes al ser humano. }\end{array}$ \\
\hline
\end{tabular}

\section{El momento de agregar valor}

En la Figura 2 se presenta las opciones para establecer el momento en el cual se puede agregar valor a un proceso productivo (Sociedad Bíblica Católica Internacional, 1972)

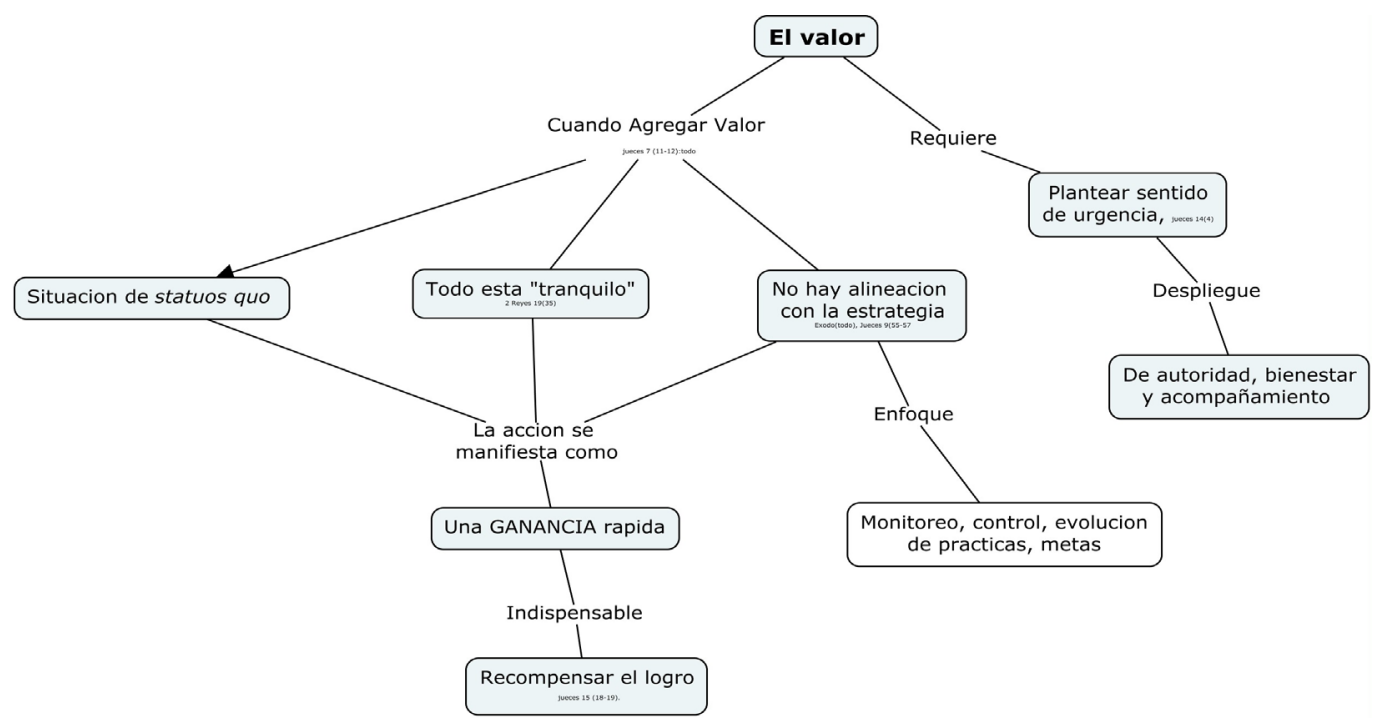

Figura 2. El momento para agregar valor 


\section{La agricultura, una oportunidad para agregar valor.}

Dado que un requisito prioritario para favorecer la agricultura, es el goce de paz. Sin duda, este es el momento oportuno para consolidar su desarrollo en Colombia. Dentro de los seis aspectos que determinaron la firma del acuerdo de paz con el grupo insurgente autodenominado las Farc, aparecen cuatro aspectos que tocan el tema de la agricultura: 1) Hacia un nuevo campo colombiano: Reforma Rural Integral; 2)Fin del conflicto; 3) Solución al problema de las drogas ilícitas; 4) Acuerdo sobre las víctimas del conflicto: "Sistema Integral de Verdad, Justicia, Reparación y No Repetición (Informe al congreso, 2017). Para tal efecto, se ha creado un mecanismo de apoyo para favorecer la restitución de tierras, la ley 1448 que igualmente se enfoca en la igualdad social mediante el desarrollo integral del campo. (Informe al congreso, 2017).

De la prospectiva mundial realizada en Kudinova et al. (2012), se destaca la agricultura y el turismo Latinoamericano, como la mejor alternativa competitiva en el mercado mundial. De esta manera. "Se dieron cuenta de que el futuro de la agricultura del mundo pasa por América del Sur: Estados Unidos consume todo lo que produce, Europa cada día es más un gran jardín y África, si bien tiene agua y tierra, no tiene todavía muchos elementos que le harían falta para ser un lugar productivo". (Terrile, 2016)

En el sector de la agricultura adicionalmente, el gobierno ha concertado dentro de los acuerdos de paz, una reforma que favorece a los agricultores y consiste en ofrecer en arriendo por un periodo de hasta 35 años las tierras del Estado para su explotación. (Balcázar, 2017).

Así mismo, al hacer una revisión del producto interno bruto (PIB) del país se encuentra que dentro de los principales renglones aportantes hasta el segundo trimestre de 2017 son, resultados comparativos respecto al mismo periodo en el 2016: 1) Agricultura, ganadería, pesca, 4,4\%. 3) Servicios sociales:(esparcimiento), servicios comunales personales, 3\%.5) comercio, reparación, restaurantes, hoteles, $0,9 \%$. 6) transporte, almacenamiento $0,7 \%$ (DANE, 2017).

Soportado en lo mencionado anteriormente, no hay duda que este es el mejor momento para volver a mirar hacia el campo. Teniendo identificadas las cinco dimensiones a través de los cuales se puede agregar valor, es el momento de identificar a cada uno de estos aspectos, dentro del campo agrícola. De esta manera, las formas de AV se encuentran inmersas en cada una de las herramientas de manufactura esbelta promulgadas hasta ahora y en los principios que favorecen la creación de valor en el ser, establecidos desde hace mucho tiempo.

Con base en lo anterior, la Tabla 2 se ha tomado, a manera de ejemplo, uno de los aspectos básicos para la agricultura y se han relacionado con esas dimensiones a través de los cuales se puede AV. (Restrepo, 2015). 
Tabla 2. Identificación de factores de Agregar Valor Caso agrícola.

\begin{tabular}{|c|c|}
\hline $\begin{array}{l}\text { Preparación de la tierra } \\
\text { (Ruiz et al., 2015) }\end{array}$ & Factor de Agregar Valor \\
\hline $\begin{array}{l}\text { Fuentes de abastecimiento hídrico, } \\
\text { heterogeneidad del relieve y configuración } \\
\text { del sistema montañoso; pendiente fuertes y } \\
\text { largas, relieve quebrado, susceptibilidad a } \\
\text { la erosión. } \\
\text { Nivel de saturación de aluminio, nivel de } \\
\text { fertilidad, comportamiento climático a través } \\
\text { del año, pisos térmicos y su impacto en la } \\
\text { temperatura y radiación solar. } \\
\text { Presión sobre el recurso y capacidad de } \\
\text { carga de los suelos. } \\
\text { Estrategias gubernamentales sobre la } \\
\text { preservación y uso de la tierra y el ambiente } \\
\text { ecológico. } \\
\text { Técnicas de cultivo; manual con maquinaria. } \\
\text { Novedades entre el saber tradicional y } \\
\text { científico (tecnología). } \\
\text { Uso de fertilizantes, rotación de cultivos, } \\
\text { renovación de pasturas, diversidad cultural } \\
\text { de la región en cuestión. } \\
\text { Uso de agroquímicos tendientes a mejorar } \\
\text { la productividad del suelo. } \\
\text { Acompañamiento institucional a todas estas } \\
\text { nuevas iniciativas. }\end{array}$ & $\begin{array}{l}\text { Características propias del proceso. } \\
\text { Mecanismos de control } \\
\text { Competencias inherentes al ser humano. }\end{array}$ \\
\hline
\end{tabular}

Respecto a los factores técnicos o productivos, se han clasificado en cuatro categorías (Daniloska, 2016): tierra y otros recursos naturales como el agua, mano de obra, capital de trabajo; maquinaria e infraestructura, administración y emprendimiento. De esta manera, se identifican algunos aspectos a través de los cuales se puede realizar un mismo ejercicio, como el descrito en el cuadro No 2, para AV: Mejoramiento de semillas y variedades en su; forma, intención, para su transporte, almacenamiento, valor de posesión que faciliten su comercio: publicidad y promoción representados en las etiquetas y marcas (Daniloska, 2016), Biotecnología para; nuevos productos en función de su valor nutricional o capacidad como fuente de energía, manipulación de la genética, sacando provecho de la manipulación de los productos para maximizar su volumen o inmunidad ante plagas y enfermedades, o bien para mejorar su resistencia hacia la falta de agua (Restrepo, 2015), factibilidad para comerciar un producto, asegurar su distribución y venta, Integración de nuevas tecnologías de transformación para crear productos más sofisticados para mercados más rentables.

Igualmente, estas oportunidades de AV se deben enfocar a aprovechar esas necesidades que están emergiendo y que se han identificado como: comportamiento social, uso de tecnología, la diversidad de economías, los medios, el cuidado de la salud, el impacto en el medio ambiente y los negocios, (Restrepo, 2015). En Flores y Flores (2013) se plantean algunos interrogantes que favorecen el diagnóstico para hacer innovación, resultados que 
están en el mismo tenor que los que aquí se han presentado.

Para finalizar, en la Figura 3, se encuentran algunos aspectos complementarios a lo que ya se han mencionado, y se resumen las principales dificultades que aparecen en la región de Boyacá, que se convierten en otras oportunidades para AV y deben ser el primer paso a dar antes de empezar a revisar el tema concreto de la agricultura (Restrepo, 2015).

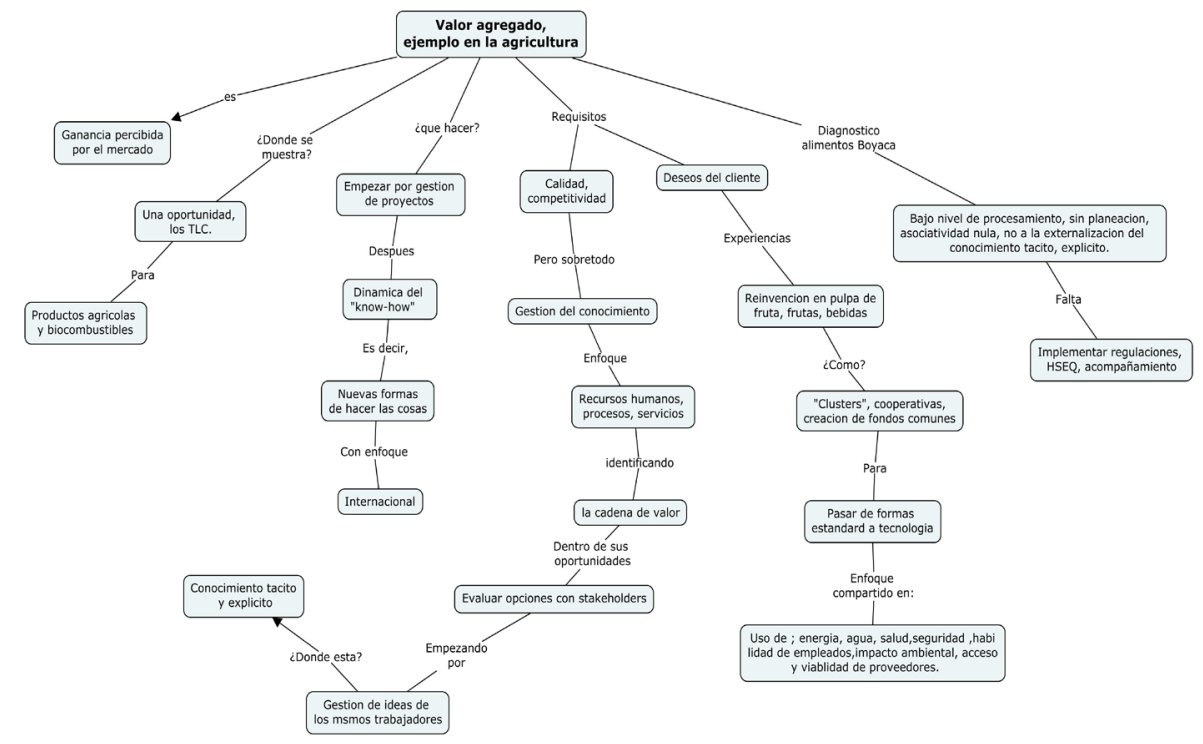

Figura 3. La agricultura en Boyacá

La realización de este documento permite orientar los esfuerzos hacia la forma de abordar el deseo por mejorar un proceso, unas características, el perfil de una persona, que podrían estar operando en condiciones donde su funcionamiento es normal, tranquilo, y quizás cumpliendo con las expectativas depositadas en él. Pero que la dinámica económica global le exige ser flexible para poder ajustarse a nuevas tendencias, experiencias, retos. Por supuesto que la humanidad ha realizado ejercicios de mejora y se han presentado en la Tabla 1 algunos de ellos. Pero se han realizado usando una metodología diferente, en cada caso, a la que se presenta en este documento y cuyo objeto es presentar una propuesta metodológica de escudriñar de manera organizada la forma de hacer mejoras. Pero no se quiere dejar de resaltar algo muy relevante y que motivo escribir el documento y es el hecho de demostrar que esas dimensiones para agregar valor han estado presentes desde hace más de 2600 años, que es la fecha de la cual data el documento de la sociedad bíblica católica internacional, de donde se han logrado extractar la información que soporta las dimensiones para AV, y solamente desde inicios de la década de los 90's, con la evolución de las estrategias de mejora propuestas para las organizaciones se ha consolidado este conocimiento.

Otro aporte de la revisión documental realizada radica en la identificación de esos momentos en los cuales es pertinente hacer el ejercicio de AV, es decir: cuando los resultados no concuerdan con lo planteado en la estrategia, las actividades se convierten en algo rutinario o cuando estas se encuentran en "aparente calma". Finalmente, y como otro valor agregado 
al trabajo, se ha aprovechado un diagnóstico de las razones por las cuales, la principal región productora de alimentos en Colombia mantiene altos niveles de pobreza en sus habitantes. Convirtiendo este diagnóstico en otra alternativa a estudiar para AV usando la metodología aquí descrita.

La determinación de las cinco dimensiones a través de las cuales se puede agregar valor en este documento, permiten enfocar las mejoras: en: un proceso, producto, organización y se podrían comparar con la propuesta realizada en Minodora (2017), quien planteo a través del diagrama propuesto Ishikawa, la forma para hacer análisis entre un problema y sus posibles causas; maquina, método, personas, mantenimiento, medio ambiente y administración. Sin embargo, el objeto del método planteado por él, pretende dividir las posibles causas de un problema dado en sus principales campos que lo pudieron generar. Para el caso objeto de estudio en este escrito, se pretende abordar cualquier actividad con el propósito de impulsar mejoras. Ahora, una vez detectadas esas mejoras, su análisis podría conducir al uso de la herramienta de Ishikawa para enfocar los recursos, esfuerzos que orienten de manera efectiva esa mejora. Así mismo, el tema puede ser enriquecido considerando reflexiones acerca de la transferencia tecnología para generar innovación, sobre la base de un diagnóstico realizado conforme como se presenta en el documento en referencia (Flores \& Flores, 2013).

\section{CONCLUSIONES}

El ejercicio realizado en este escrito se convierte en una forma de enfocar, disgregar las mejoras en un proceso de transformación de un bien o servicio, muy pertinente para el caso Latinoamericano y específicamente el colombiano, ya que la agricultura emerge como la mejor opción económica de desarrollo, y así lo ha considerado el senado de la república, después de que dispuso en el plan nacional de desarrollo las condiciones para propiciar su crecimiento.

\section{AGRADECIMIENTOS}

Al grupo de investigación Gridse, adscrito al programa de ingeniería electromecánica de la Universidad Pedagógica y Tecnológica de Colombia, seccional Duitama, Boyacá, Colombia por el apoyo a la financiación de este proyecto. 


\section{REFERENCIAS}

BALCAZAR, C. Atacar a Santos es abrir paso a los populismos. El tiempo. 1 de septiembre de. 13p. 2017. Bogotá. Colombia

BALLEN, M. 2007. Teorías, procesos. Segunda edición. Bogotá. Colombia: Universidad Cooperativa de Colombia, 2007. ISBN: 958-8325-24-8.

CALDERON, G. Aproximación a un modelo de gestión humana que agregue valor a la empresa colombiana. [en línea]. Primera edición. Manizales. Colombia: Universidad Nacional de Colombia, 2008. Disponible en: http://bdigital.unal.edu.co/5067/2/ gregoriocalderonhernandez.2008.pdf

CHACON, M. y GARCIA, R. (2015). Heurística para el balanceo de líneas de ensamble. Revista Ingeniería Industrial [en línea]. 14(1), 23-35. Disponible en: http://revistas.ubiobio.cl/ index.php/RI/article/view/1913

CHÁVEZ PORRAS, Á. y GUARÍN CORTÉS, N. (2014). Gestión logística y operacional de la unidad de reciclaje de residuos de construcción y demolición. Revista Ingeniería Industrial [en línea]. 13(2),7-18. Disponible en: http://revistas.ubiobio.cl/index.php/Rl/article/view/89

CHYBALSKI, F. (2017). Forecasts valúes added (FVA) analysis as a means to improve the efficiency of a forecasting process. Operations Research \& Decisions [en línea]. 27(1), 5-19. Doi: 10.5277/ord170101. Disponible en: https://doi.org/10.5277/ord170101

DANE. Producto interno bruto II trimestre. En DANE [en línea]. [Consulta: 15-julio-2017]. Disponible en:http://www.dane.gov.co/index.php/52-espanol/noticias/noticias/4282-productointerno-bruto-pib-ii-trimestre-2017.

DANILOSKA, N. (2016). Concept of values-added in agriculture as a responde to competitive marketplace. Economic Development / Ekonomiski Razvoj. [en línea].18(1/2), 115-124. Disponible en: https://www.ceeol.com/search/article-detail?id=410977

DELGADO, J. Pertinencia académica del principal motor de búsqueda, google: Una propuesta para el docente. En: I er encuentro internacional de investigación universitaria. [En línea]. Tunja. Colombia. 2017a. Disponible en: https://repository.usta.edu.co/handle/11634/17684

DELGADO, J. Pobreza: una oportunidad de salida. CIDEA (coordinación evento). "Los Objetivos de Desarrollo Sostenible ODS. En: III encuentro internacional de educación a distancia y virtual. Tunja. Colombia. 2017b

DELGADO, J. El perfil del trabajador auto controlado. Inquietud empresarial [en línea]. 15(1), 78-103. 2015. Disponible en: https://revistas.uptc.edu.co/index.php/inquietud_ empresarial/article/view/3920

DRUSKAT, V. U. y WHEELER, J. V. (2004). How to Lead a Self-Managing Team? MIT Sloan Management. Review [en línea]. 45(4), 65-71. Disponible en:

http://search.proquest.com/openview/fce30d878acaa6271e5be92fa82b0314/1?pqorigsite $=$ gscholar $\& \mathrm{cbl}=26142$ 
GONZALEZ, C. F. (2017). Manufactura esbelta (Lean manufacturing principales herramientas). Revista Panorama Administrativo [en línea]. Año 2017. 1(2). Disponible en: http://www. itcelaya.edu.mx/ojs/index.php/raites/article/view/77

INFORME AL CONGRESO. Cap. 1: Política macroeconómica. 2017, pp.13-29. [en línea]. Disponible en: https://colaboracion.dnp.gov.co/CDT/Sinergia/Documentos/Informe\%20al\%20 Congreso\%20Presidencia\%202017_Baja_f.pdf.

KUDINOVA, G., ROZENBERG, G. y YURINA, V. (2012). Towards a "green" economy: The way to sustainable development and poverty eradication. Nai-robi (Kenya), Geneva (Switzerland), Moscow (Russia): UNEP, 2011. Principles of the Ecology [en línea]. 1(4), 41-48. Disponible en: http://ecopri.ru/files/pdfs_a4T6sdq42RtE34werSR/article_1602.pdf

MINODORA, L. y ALIN, S. (2017). Study to determine a new model of the ishikawa diagram for quality improvement. Fiability \& Durability [en línea]. 1(19), 249-254.

LIKER, J. The Toyota Way. New York, U.S.A: McGraw Hill. 2004.

MOBLEY, R.K. PROCESS AND VALUE-STREAM MAPPING. En: Maintenance Engineering Handbook. 8 Edition, Chapter McGraw-Hill Professional, 2014. ISBN: 9780071826617

FLORES, P. y FLORES, M. (2013). El Comportamiento Innovador en Valor Agregado del Sector Agrícola en el Estado de Sinaloa. Journal of technology management \& innovation [en línea]. 8, 140-150. Disponible en: http://dx.doi.org/10.4067/S0718-27242013000300042

PORTER, M. (2008). The five competitive forces that shape strategy. Harvard business review [en línea]. 86(1), 78-93. Disponible en: https://media.mises.org/Academy/2014_ winter/managers/HBR_on_Strategy.pdf\#page=25

RAJADELL, M. y SANCHEZ, J. Lean manufacturing: La evidencia de una necesidad. Ediciones Díaz de Santos: Madrid. España. 2010. ISBN 9788479789671

RESTREPO, S. A. Desarrollo de productos de alto valor agregado como estrategia exportadora para las pymes del sector agroindustrial de Boyacá. Desde la perspectiva de gestión de proyectos.[en línea]. Trabajo de maestría. Universidad Nacional De Colombia. Trabajo de maestría. Bogotá. Colombia. 2015. Disponible en: http://revistas.ustatunja.edu.co/ index.php/ivestigium/article/view/1006/1016

SOCIEDAD BÍBLICA CATÓLICA INTERNACIONAL.1972. Éxodo, (todo), Jueces 2(1-5): 7(11-12):9(55-57):14(4): 15(18-19),, 1 Corintios 1281-12), Numero 11 (15-17), Génesis 39, (2-6), Apocalipsis 412), Reyes 17(25-28), Isaías 11 (1-16): 65(13-25)., 1 Pedro 1 (3-6), Ed. Verbo divino. Navarra, España.

TEOFILO, P. (2016). Una Asociación con valor agregado: La Pertenencia. Revista de la Asociación Argentina de Ortopedia y Traumatología [en línea]. 81(1). Disponible en: http:// dx.doi.org/10.15417/579

TERRILE, S. Agregar valor: frente al desafío de responder al reto de alimentar al mundo. La Nación, 16 de julio 2017. Disponible en: http://www.lanacion.com.ar/1918571-agregar-valorfrente-al-desafio-de-responder-al-reto-de-alimentar-al-mundo 